\title{
Starch accumulation in the lungs of two infants following positive ventilation
}

\author{
Kana Unuma $\cdot$ Kazuki Harada $\cdot$ Teruaki Oka $\cdot$ \\ Koichi Uemura
}

Accepted: 25 April 2013/Published online: 23 May 2013

(C) The Author(s) 2013. This article is published with open access at Springerlink.com

\begin{abstract}
Two infants in different nurseries were found with cardiopulmonary arrest. Cardiopulmonary resuscitation was undertaken immediately in both cases, but was unsuccessful. The cause of death in both infants was diagnosed as sudden unexpected death, probably sudden infant death syndrome, at postmortem investigations. Microscopic examination at autopsy showed the presence of starch granules in the lungs. These were probably introduced during tracheal or nasotracheal intubation for cardiopulmonary resuscitation from gloves sterilized with powdered cornstarch. In both cases cellular staining of foreign bodies was weak and there were few starch granules within macrophages. Our findings suggest that the detection of cornstarch in the lungs can be an artifact arising from surgical gloves used in resuscitation. This artifact may easily occur in infants because of their immature lungs and short respiratory tract. Non-powdered gloves should be worn instead of powdered gloves during tracheal intubation, especially in infants.
\end{abstract}

Keywords Postmortem - Lungs · Starch · Autopsy · Artifact

K. Unuma $(\varangle) \cdot$ K. Uemura

Department of Forensic Medicine, Graduate School of Medical and Dental Sciences, Tokyo Medical and Dental University, 1-5-45 Yushima, Bunkyo-ku, Tokyo 113-8519, Japan e-mail: unumlegm@tmd.ac.jp

K. Harada

Department of Forensic Medicine, National Defense Medical College, 3-2 Namiki Tokorozawa, Saitama 359-8513, Japan

T. Oka

Division of Pathology, Kanto Central Hospital, 6-25-1

Kamiyouga, Setagaya-ku, Tokyo 158-8531, Japan

\section{Introduction}

Accidental cornstarch aspiration may occur from surgical gloves powdered with cornstarch. Dain et al. [1,2] and Milton described starch in the lungs of newborns after the use of positive pressure ventilation, but to the best of our knowledge, there have been no subsequent reports of cases of accidental cornstarch aspiration.

Common surgical glove powder is made of cornstarch, which is easily stripped from the gloves or hands. Powdered gloves contain $130-250 \mathrm{mg}$ of starch per pair, even after washing and air drying [3, 4]. Histologically, surgical glove powder appears as round or polyhedral bodies, varying between 10 and $70 \mu \mathrm{m}$ in diameter, which stain faintly basophilic with hematoxylin and eosin (H\&E) staining, and bright red with periodic acid-Schiff (PAS) staining [1]. A typical Maltese cross is seen under polarized light.

We describe two cases of infant death with cardiopulmonary arrest in different nurseries. Cardiopulmonary resuscitation (CPR) was unsuccessful in both cases. The cause of death in the two infants was diagnosed as sudden infant death syndrome (SIDS). Furthermore, both infants had starch granules in their lungs. More than $99 \%$ of the starch granules were embedded in the bronchial and alveolar regions without alveolar macrophages, indicating that the particles were of postmortem origin. In both cases, surgical gloves powdered with cornstarch were used during attempted CPR at an emergency hospital.

These findings suggest that cornstarch in the lungs can be an artifact arising from surgical gloves that are put on carelessly, and this artifact may easily occur in infants because of their immature lungs and a short respiratory tract. 


\section{Case report}

We describe two cases of sudden death in a 10-month-old male infant and an 8-month-old female infant. Both infants were products of uneventful pregnancies and normal, spontaneous, vaginal deliveries, and in good health until the day of death.

The postmortem investigations for each infant fulfilled most of the criteria corresponding to the San Diego definition of SIDS. Screening for congenital metabolic disorders in the newborn infants were negative. A full-body X-ray and computed tomography (CT) showed no fractures. Toxicological analyses of the blood, urine, and gastric contents revealed no drugs. Bacterial and viral cultures of the blood were negative. C-reactive protein (CRP), an index of inflammation, was negative. The postmortem investigations diagnosed the cause of death in the two infants as sudden unexpected death, probably SIDS.

Case 1: The first infant was a 10-month-old male who died on a cold winter day in a nursery. He was fed milk and cookies at approximately 10:00 a.m. At approximately 11:00 a.m., he was placed together with two other infants in the prone position in the middle of a large baby bed covered with a fitted bed sheet and a blanket that was usually kept in a bedroom. Two school staff were in the living room performing work on a computer until approximately 11:30 a.m. One of the staff members checked on the infant in bed at this time and found him unresponsive. CPR was performed immediately and he was taken to the emergency room in a hospital, but resuscitation was unsuccessful. He suffered from ventricular fibrillation in the ambulance and from cardiac arrest after arriving at the hospital.

A complete judicial autopsy examination showed an anatomically normal infant. The infant appeared adequately developed and well nourished for his age; he weighed 9,500 $\mathrm{g}$ and was $76 \mathrm{~cm}$ in length. There was no evidence of trauma. All internal viscera were unremarkable, except for visceral congestion, including pulmonary (lung weight, left/right: 75/90 g) and cerebral edema (brain weight, $930 \mathrm{~g}$ ), and congestion.

Case 2: The second infant was an 8-month-old female who died on an early summer day in a nursery. This infant and nursery had no known direct familial link or relationship to Case 1. She was fed approximately $200 \mathrm{~mL}$ of milk at approximately $12: 40$ p.m. She was put to bed in the supine position with her head turned sideways at approximately 1:35 p.m. and was heard to cry once at 2:45 p.m. The school's staff were in the same room playing with the infant's 2-year-old brother until approximately 4:15 p.m. The staff checked on the infant in bed at this time and found her unresponsive. CPR was undertaken immediately, but death was certified after $1.5 \mathrm{~h}$.
A complete judicial autopsy examination showed an anatomically normal infant. The baby appeared adequately developed and well nourished for her age; she weighed $7,500 \mathrm{~g}$ and was $70 \mathrm{~cm}$ in length. An autopsy revealed no evidence of trauma. All internal viscera were unremarkable, except for visceral congestion including pulmonary (lung weight, left/right: 60/70 g) and cerebral edema (brain weight: $900 \mathrm{~g})$.

\section{Microscopy}

Histologically, round to polyhedral bodies varying in size between 5 and $60 \mu \mathrm{m}$ were stained faintly basophilic with H\&E (Fig. 1a) and bright red with PAS staining (Fig. 1c). These bodies were found in bronchial and alveolar tissue in both cases. The findings of relatively large bodies in bronchi and relatively small bodies in alveoli indicated introduction of foreign bodies. A typical Maltese cross was seen under polarized light (Fig. 1b). More than $99 \%$ of the starch granules were embedded in the bronchial and alveolar region without a response of alveolar macrophages, indicating that the particles were of postmortem origin.

To confirm that the foreign bodies observed in the infants' lungs were derived from cornstarch on surgical gloves, a suspension of cornstarch used on non-powdered gloves (Fig. 2a) and powdered gloves (Fig. 2b) was examined using similar staining. We observed a Maltese cross pattern and the cornstarch crystals had the same morphology as the particles in the lungs of gloves with starch (Fig. 2b). Starch granules were not found in the lungs in any of 16 SIDS infants who had undergone CPR with non-powdered gloves (data not shown). Only one of 30 adult autopsy cases who had undergone CPR with powdered gloves revealed one cornstarch granule in 10 fields (data not shown).

\section{Discussion}

We experienced two cases of infants with starch in the lungs at autopsy. The cause of death in both infants was diagnosed as sudden unexpected death, probably SIDS, at postmortem investigations.

Several starch granules were present within bronchial and alveolar tissue in both infants. However, few starch granules were observed in adults who had undergone CPR with powder gloves. These results indicate that there might be more cases of infants with starch granules following resuscitation than adults, and this might be due to the immature lungs and short respiratory tract of infants. The lungs of a newborn contain approximately 20 million alveoli, which then grow to 300 million, and increase in 

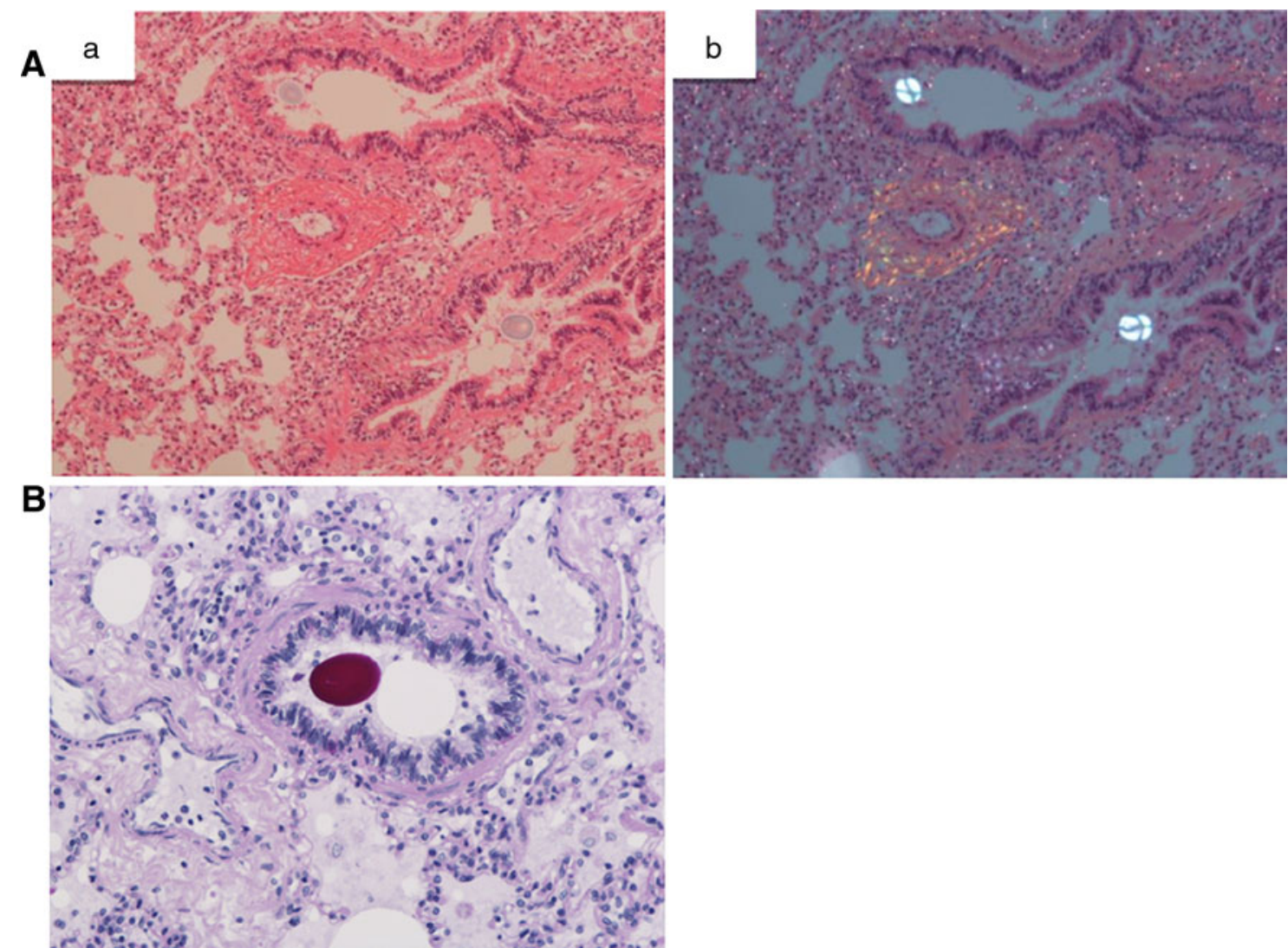

Fig. 1 Starch granules embedded in bronchi and alveoli. (a) Panel $A-a$ : Starch granules are stained faintly basophilic with hematoxylin and eosin $(\mathrm{H} \& \mathrm{E}, \times 100)$. (b) Panel A-b: A typical Maltese cross is seen under polarized light. (c) Panel B: Starch granules are stained bright $r e d$ with periodic acid-Schiff (PAS) staining in bronchial tissue $(\times 200)$
Fig. 2 Starch granules from a surgical glove that was nonpowdered (a) or powdered (b) $(\times 200$; insert, $\times 1,000)$
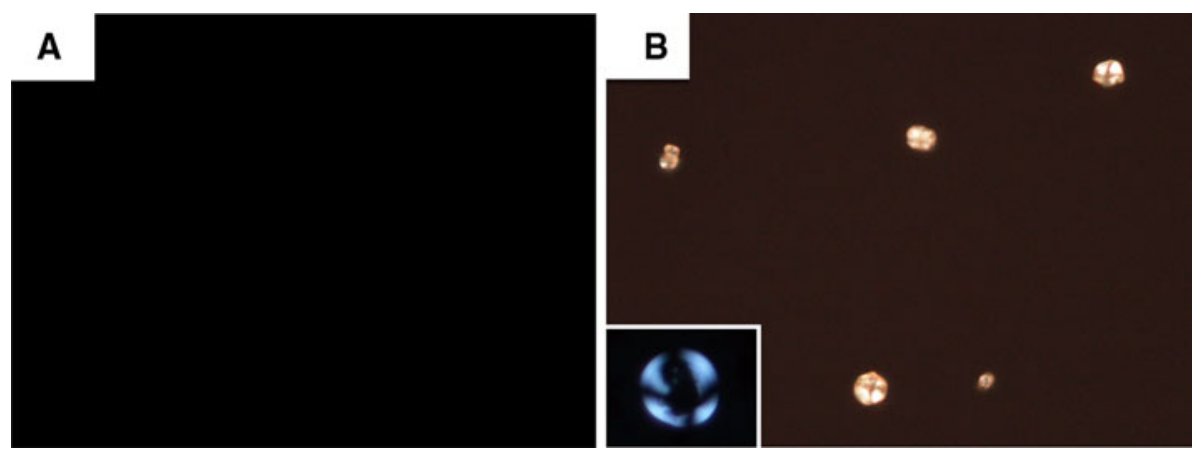

size as the child becomes an adult [5]. In children, short and immature bronchi and fewer alveoli may facilitate deeper aspiration of foreign bodies into the alveoli. In addition, gloves opened over an intubation tube or a carelessly attached intubation tube may cause this artifact.

Acute aspiration of zinc, copper, or talc powder is dangerous in children, and several fatalities have been reported [6-8]. However, cornstarch aspiration has not been considered as a cause of death, although Silver et al. [9] reported that careless use of cornstarch powder for infants could lead to respiratory failure. To the best of our knowledge, this previous report is the only report of a fatality related to cornstarch aspiration. In this report the diagnosis was uncertain based on clinical findings because the finding of cornstarch in broncho-alveolar lavage was used to diagnose the cause of death as respiratory dysfunction due to severe pulmonary inflammation [9]. In our cases neither infant had symptoms of respiratory dysfunction.

In our two infants the round or polyhedral bodies found in their bronchial and alveolar tissue were histologically shown to be starch granules. The powder attached to the inside of surgical gloves, which are used during CPR at an emergency room in a hospital, was also investigated. We 
found that the crystals of the glove powder had the same morphology as the starch granules found in the lungs. In addition, starch granules were not found in the lungs of SIDS infants who had undergone CPR with powder-free gloves. Therefore, we concluded that the granules were probably introduced into the lungs of the two infants from sterile powdered gloves during tracheal or nasotracheal intubation. More than $99 \%$ of the starch granules were found without macrophages, which suggests that the particles were of postmortem origin.

In conclusion, we present two new findings. First, cornstarch in bronchi and alveoli may occur artificially because of introduction during CPR from surgical glove powder. Second, this artifact may easily occur in infants because of their immature lungs and short respiratory tract. Nonpowdered gloves should be worn instead of powdered gloves during tracheal intubation, especially in infants. Forensic pathologists should not consider that cornstarch in the bronchi and alveoli has occurred during life as it may lead to an incorrect conclusion regarding the cause of death.

\section{Key Points}

1. Findings from the presented cases suggest that cornstarch in the lungs can be an artifact arising from the use of powdered gloves during tracheal intubation.

2. This artifact may occur in infants because of their immature lungs and short respiratory tract.

3. The gloves that are used during tracheal intubation should be non-powdered to prevent the introduction of powder into the airway, especially in infants.
4. Forensic pathologists should not consider that cornstarch in the bronchi and alveoli has occurred during life as it may lead to an incorrect conclusion regarding the cause of death.

Open Access This article is distributed under the terms of the Creative Commons Attribution License which permits any use, distribution, and reproduction in any medium, provided the original author(s) and the source are credited.

\section{References}

1. Dain DW, Randall JL, Smith JW. Starch in the lungs of newborns following positive pressure ventilation. Am J Dis Child. 1970;119:218-20.

2. Finegold MJ. Starch in lungs of newborn. Am J Dis Child. 1981;135:671-2.

3. Lee CM, Lehman EP. Experiments with non-irritating glove powder. Surg Gynec Obstet. 1947;84:689-95.

4. McNaught GHD. Starch granuloma: a present day surgical hazard. Br J Surg. 1964;51:845-9.

5. Reid L. Lung growth in health and disease. $\mathrm{Br} \mathrm{J}$ Dis Chest. 1984;78:113-34.

6. Pfenninger J, D'Apuzzo V. Powder aspiration in children. Report of two cases. Arch Dis Child. 1977;52:157-9.

7. Molnar JJ, Nathenson G, Edberg S. Fatal aspiration of talcum powder by a child. Report of a case. N Engl J Med. 1962; 266:36-7.

8. Donoso A, Cruces P, Camacho J, Ríos JC, Paris E, Mieres JJ. Acute respiratory distress syndrome resulting from inhalation of powdered copper. Clin Toxicol. 2007;45:714-6.

9. Silver P, Sagy M, Rubin L. Respiratory failure from corn starch aspiration: a hazard of diaper changing. Ped Emerg Care. 1996; $12: 108-10$. 